\title{
Light baryon spectrum using improved interpolating operators
}

\author{
Lattice Hadron Physics Collaboration (LHPC): S. Basak, ${ }^{a}$ R. G. Edwards, ${ }^{b}$ G. T. \\ Fleming ${ }_{,}^{c}$ J. Juge, ${ }^{d}$ A. Lichtl, ${ }^{d}$ C. Morningstar ${ }^{d}$ D. G. Richards,${ }^{b}$ I. Sato ${ }^{* e}$, S. J. \\ Wallace, ${ }^{f}$ \\ ${ }^{a}$ Department of Physics, N.N.D. College, Calcutta 700092, India \\ ${ }^{b}$ Thomas Jefferson National Accelerator Facility, Newport News, VA 23606, USA \\ ${ }^{c}$ Yale University, New Haven, CT 06520, USA \\ ${ }^{d}$ Department of Physics, Carnegie Mellon University, Pittsburgh, PA 15213, USA \\ ${ }^{e}$ Lawrence Berkeley Laboratory, Berkeley, CA 94720, USA \\ ${ }^{f}$ Department of Physics, University of Maryland, College Park, MD 20742, USA
}

Energies for excited light baryons are computed in quenched QCD with a pion mass of $490 \mathrm{MeV}$. Operators used in the simulations include local operators and the simplest nonlocal operators that have nontrivial orbital structures. All operators are designed with the use of Clebsch-Gordan coefficients of the octahedral group so that they transform irreducibly under the group rotations. Matrices of correlation functions are computed for each irreducible representation, and then the variational method is applied to separate mass eigenstates. We obtained 17 states for isospin $1 / 2$ and 11 states for isospin $3 / 2$ in various spin-parity channels including $J^{P}=5 / 2^{ \pm}$. The pattern of the lowest-lying energies from each irrep is discussed. We use anisotropic lattices of volume $24^{3} \times 64$ with temporal lattice spacing $a_{t}^{-1}=6.05 \mathrm{GeV}$ with renormalized anisotropy $\xi=3.0$.

XXIVth International Symposium on Lattice Field Theory

July 23-28, 2006

Tucson, Arizona, USA

\footnotetext{
${ }^{*}$ Speaker.
} 


\section{Introduction}

The theoretical determination of the baryon spectrum is an important step for lattice QCD. Lattice QCD results have provided reasonable agreement with the physical ground state masses of different baryons (for a review, see [1]). For excited states of baryons, several groups have reported results [2-7], but further improvements are needed for more precise determinations of the masses and spins for excited particles. In this report, we show preliminary results for nucleon and delta baryon masses including $J^{P}=5 / 2^{ \pm}$using improved lattice baryon fields with quenched anisotropic lattices.

Spins of states on a cubic lattice are deduced from the level orderings of energies computed in different irreducible representations (irreps) of the octahedral group. Source and sink operators have to be designed so as to transform as a definite irrep of the octahedral group in order to identify a total angular momentum of a continuum state. There are six double-valued irreps of the octahedral group: $G_{1 g}, H_{g}, G_{2 g}, G_{1 u}, H_{u}$ and $G_{2 u}$, in which "g" subscript denotes positive parity (gerade) and "u" subscript denotes negative parity (ungerade). Subduction pattern of total angular momentum to octahedral irreps is summarized in Table 1.

In general, a lattice operator with a definite irrep couples to multiple states corresponding to different total angular momenta according to the subduction pattern. To separate the individual energy eigenstates included in an irrep, we employ the variational technique, which involves diagonalization of a matrix of correlation functions. Substantial numbers of basis operators are needed in each channel in order to provide sufficiently large degrees of freedom for the determination of the spectrum of excited states. Inclusion of multi-hadron operators in the construction of matrices of correlation functions is needed to separate the resonances from the hadron scattering states. In this work, we only used three-quark operators. Thus the separation of discrete scattering states and single-hadron states in the excited spectrum is beyond scope of this work, and we refer to our lattice results as "energies" rather than as "masses".

\begin{tabular}{|llllllll|}
\hline$\Lambda$ & $\mathrm{J}=$ & $\frac{1}{2}$ & $\frac{3}{2}$ & $\frac{5}{2}$ & $\frac{7}{2}$ & $\frac{9}{2}$ & $\frac{11}{2}$ \\
$G_{1}$ & & 1 & 0 & 0 & 1 & 1 & 1 \\
$H$ & & 0 & 1 & 1 & 1 & 2 & 2 \\
$G_{2}$ & & 0 & 0 & 1 & 1 & 0 & 1 \\
\hline
\end{tabular}

Table 1: The number of occurrences of double-valued irrep $\Lambda=\left\{G_{1}, H, G_{2}\right\}$ of the octahedral group for different values of continuum $J$ (up to $11 / 2$ ).

\section{Improved baryon operators}

We construct operators that transform according to each irrep of the octahedral group by employing the analytical method based on appropriate Clebsch-Gordan coefficients for the group [8]. An alternative method of constructing vast numbers of operators in an automated procedure is presented in Ref. [9]. We used complete sets of quasi-local and one-link three-quark operators in this work. 
Quasi-local baryon operators consist of smeared quark fields $\widetilde{q}(\mathbf{x}, t)$ that are located at the same space point at which the color singlet contraction is taken,

$$
\bar{\Psi}_{\Lambda, \lambda, m}^{(I S)}(\mathbf{x}, t)=\varepsilon_{i j k} f_{a b c}^{(I S)} c_{\alpha \beta \gamma}^{(\Lambda, \lambda, m)} \overline{\widetilde{q}}_{\alpha}^{i, a}(\mathbf{x}, t) \overline{\widetilde{q}}_{\beta}^{j, b}(\mathbf{x}, t) \overline{\widetilde{q}}_{\gamma}^{k, c}(\mathbf{x}, t),
$$

where $i, j, k$ are color indices, $a, b, c$ are flavor indices, and $\alpha, \beta, \gamma$ are Dirac indices. Octahedral group irreps are denoted by $\Lambda=\left\{G_{1 g}, H_{g}, G_{2 g}, G_{1 u}, H_{u}, G_{2 u}\right\}$. The corresponding dimensions $d_{\Lambda}$ are $2,4,2,2,4,2$. The rows of an irrep are distinguished by row labels $\lambda=1, \cdots, d_{\Lambda}$. When there are more than one operators in a single $(\Lambda, \lambda)$ channel, we use the additional index $m$ to label the different operators. These labels are the indices of matrices of correlation functions when source and sink operators from a given irrep are used. Isospin $I$ and strangeness $S$ are selected by coefficients $f_{a b c}^{(I S)}$. We use the mass for up and down quarks, and do not consider strange quarks in this work. The explicit Clebsch-Gordan coefficients $c_{\alpha \beta \gamma}^{(\Lambda, \lambda, m)}$ are given in Ref. [8].

A simple extension of quasi-local operators is to displace one of the quark fields along a spatial axis and to include a straight gauge-link so as to keep the gauge covariance. The displacement operator $\hat{d}_{l}$ acted on three-quark operator is defined as follows,

$$
\hat{d}_{l} \bar{\Psi}_{\Lambda, \lambda, k}^{(I S)}(\mathbf{x}, t)=\varepsilon_{i j k} f_{a b c}^{(I S)} c_{\alpha \beta \gamma}^{(\Lambda, \lambda, k)} \overline{\tilde{q}}_{\alpha}^{i, a}(\mathbf{x}, t) \overline{\tilde{q}}_{\beta}^{j, b}(\mathbf{x}, t) \overline{\tilde{q}}_{\gamma}^{k^{\prime}, c}\left(\mathbf{x}+a_{s} \hat{l}, t\right) U_{l}^{\dagger k^{\prime} k}(\mathbf{x}, t),
$$

where $\hat{l}$ takes six spatial directions, $\pm \hat{x}, \pm \hat{y}, \pm \hat{z}$. Assuming that lattice gauge links recover the cubic symmetry with large statistics, displacement operators transform amongst themselves under lattice rotations. By having the following similarity transformed bases,

$$
\left(\begin{array}{c}
\hat{A}_{1} \bar{\psi} \\
\hat{D}_{+} \bar{\psi} \\
\hat{D}_{-} \bar{\psi} \\
\hat{D}_{0} \bar{\psi} \\
\hat{E}_{0} \bar{\psi} \\
\hat{E}_{2} \bar{\psi}
\end{array}\right) \equiv\left(\begin{array}{c}
\frac{1}{\sqrt{6}}\left(\hat{d}_{x} \bar{\psi}+\hat{d}_{y} \bar{\psi}+\hat{d}_{z} \bar{\psi}+\hat{d}_{-x} \bar{\psi}+\hat{d}_{-y} \bar{\psi}+\hat{d}_{-z} \bar{\psi}\right) \\
\frac{i}{2}\left[\left(\hat{d}_{x} \bar{\psi}-\hat{d}_{-x} \bar{\psi}\right)+i\left(\hat{d}_{y} \bar{\psi}-\hat{d}_{-y} \bar{\psi}\right)\right] \\
-\frac{i}{2}\left[\left(\hat{d}_{x} \bar{\psi}-\hat{d}_{-x} \bar{\psi}\right)-i\left(\hat{d}_{y} \bar{\psi}-\hat{d}_{-y} \bar{\psi}\right)\right] \\
-\frac{i}{\sqrt{2}}\left(\hat{d}_{z} \bar{\psi}-\hat{d}_{-z} \bar{\psi}\right) \\
\frac{1}{\sqrt{12}}\left[2\left(\hat{d}_{z} \bar{\psi}+\hat{d}_{-z} \bar{\psi}\right)-\left(\hat{d}_{x} \bar{\psi}+\hat{d}_{-x} \bar{\psi}\right)-\left(\hat{d}_{y} \bar{\psi}+\hat{d}_{-y} \bar{\psi}\right)\right] \\
\frac{1}{2}\left[\left(\hat{d}_{x} \bar{\psi}+\hat{d}_{-x} \bar{\psi}\right)-\left(\hat{d}_{y} \bar{\psi}+\hat{d}_{-y} \bar{\psi}\right)\right]
\end{array}\right)
$$

the displacements $\hat{A}_{1}, \hat{D}_{ \pm, 0}$, and $\hat{E}_{0,2}$ transform according to $A_{1}, T_{1}$, and $E$ irreps of the octahedral group, respectively. Here $A_{1}$ is the cubically symmetric, one-dimensional irrep, $T_{1}$ is the threedimensional irrep that corresponds to $\ell=1$ and $E$ is the two-dimensional irrep that corresponds to $\ell=2$ for the lowest orbital angular momentum $\ell$. These new bases are chosen so that their transformations resemble those of the spherical harmonics $Y_{\ell m}$, i.e., $\hat{A}_{1} \sim Y_{00}, \hat{D}_{+, 0,-} \sim Y_{11}, Y_{10}, Y_{1-1}$, and $\hat{E}_{0,2} \sim Y_{20},\left(Y_{22}+Y_{2-2}\right)$. The $A_{1}, T_{1}$, and $E$ one-link operators are now defined as

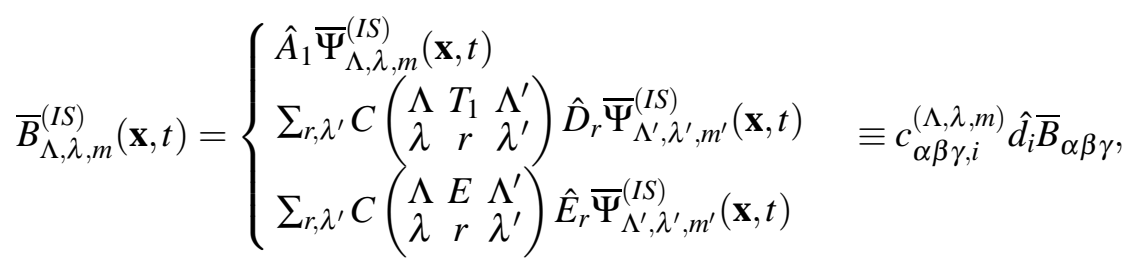

where $C\left(\begin{array}{lll}\Lambda & \Lambda^{\prime} & \Lambda^{\prime \prime} \\ \lambda & \lambda^{\prime} & \lambda^{\prime \prime}\end{array}\right)$ are the Clebsch-Gordan coefficients that takes the direct product of irreps $\Lambda^{\prime}$ and $\Lambda^{\prime \prime}$ to yield overall irrep $\Lambda[8]$. 
We then use improved operators to construct matrices of correlation functions

$$
C_{m m^{\prime}}^{(\Lambda \lambda)}(t) \delta_{\Lambda \Lambda^{\prime}} \delta_{\lambda \lambda^{\prime}}=c_{\alpha \beta \gamma, i}^{(\Lambda \lambda m) *} c_{\mu v \rho, i^{\prime}}^{\left(\Lambda^{\prime} \lambda^{\prime} m^{\prime}\right)} \sum_{\mathbf{x}}\left\langle 0\left|B_{\alpha \beta \gamma}(\mathbf{x}, t) \hat{d}_{i}^{\dagger} \hat{d}_{i^{\prime}} \bar{B}_{\alpha^{\prime} \beta} \gamma^{\prime}(\mathbf{0}, 0)\right| 0\right\rangle \gamma_{\alpha^{\prime} \mu}^{4} \gamma_{\beta^{\prime} v}^{4} \gamma_{\gamma^{\prime} \rho}^{4},
$$

where a displacement operator $\hat{d}_{i}$ applied to a baryon field denotes a one-link baryon operator for $i= \pm x, \pm y, \pm z$ and a quasi-local baryon for $i=0$. Lattice operators belonging to different irreps or to different rows of one irrep are orthogonal because of the octahedral symmetry of the lattice. Different embeddings of a given irrep and row provide operators appropriate to forming a matrix of correlation functions, to which the variational method is applied in order to determine the spectrum of states. Three Dirac $\gamma_{4}$ matrices are included in Eq. (2.5) in order to produce a Hermitian matrix of correlation functions.

\section{Computational methods}

\subsection{Variational method}

Analyses of excited state energies are based upon the matrices of correlation functions of Eq. (2.5). In order to extract the spectrum of energies from the matrix of correlation functions, we first average over rows because they provide equivalent results owing to octahedral symmetry. We then solve the following generalized eigenvalue equation,

$$
\sum_{k^{\prime}} \widetilde{C}_{k k^{\prime}}^{(\Lambda)}(t) v_{k^{\prime}}^{(n)}\left(t, t_{0}\right)=\alpha^{(n)}\left(t, t_{0}\right) \sum_{k^{\prime}} \widetilde{C}_{k k^{\prime}}^{(\Lambda)}\left(t_{0}\right) v_{k^{\prime}}^{(n)}\left(t, t_{0}\right),
$$

where superscript $n$ labels the eigenstates. The symbol $\widetilde{C}^{(\Lambda)}$ indicates that the appropriate average over rows has been performed. The reference time $t_{0}$ in Eq. (3.1) is taken near the source time $t=0$ in order to have significant contributions from excited states. The generalized eigenvalues $\alpha^{(n)}\left(t, t_{0}\right)$ are related to the energy $E_{n}$ by [10]

$$
\alpha^{(n)}\left(t, t_{0}\right) \simeq e^{-E_{n}\left(t-t_{0}\right)}\left[1+O\left(e^{-|\delta E| t}\right)\right],
$$

where $\delta E$ is the difference between $E_{n}$ and the next closest energy. We have determined effective energies $E_{n}$ by fitting the generalized eigenvalues to the leading term of Eq. (3.2).

\subsection{Lattice action}

We employed anisotropic lattices with volume $24^{3} \times 64$, where the temporal lattice spacing $a_{t}$ is three times finer than the spatial lattice spacing $a_{s}$. Gauge configurations are generated using the quenched, anisotropic, unimproved Wilson gauge action [11] with $\beta=6.1$ and the pion mass is $490 \mathrm{MeV}$. A total of 167 configurations is used, but statistical ensembles are effectively doubled by use of CPT symmetry. The scale is $a_{t}^{-1}=6.05 \mathrm{Gev}$ in these lattice, provided by the analysis of string tension.

\section{Results and discussions}

\subsection{Effective energies}

We have extracted energies for isospin $1 / 2$ and $3 / 2$ channels by diagonalizing matrices of correlation functions formed from three-quark operators that share the same octahedral symmetry. 
We diagonalize matrices of different dimensions by adding or subtracting trial operators to find optimal sets of operators. Figure 1 shows low-lying effective energies as a function of matrix dimensions. Energies are determined from single-exponential fits to the generalized eigenvalues. We found that low-lying energy states are fairly stable with respect to the number of operators used, as long as a few important operators are included. It is expected that use of a larger number of operators should improves the low-lying spectrum because contaminations from higher-lying states are reduced. This expectation appears to hold except for a few excited states that are sensitive to the types of operators used. The lowest-lying effective energies in the $G_{1 g}, H_{g}, G_{2 g}$ channels with isospin 1/2 are plotted in Fig. 2.

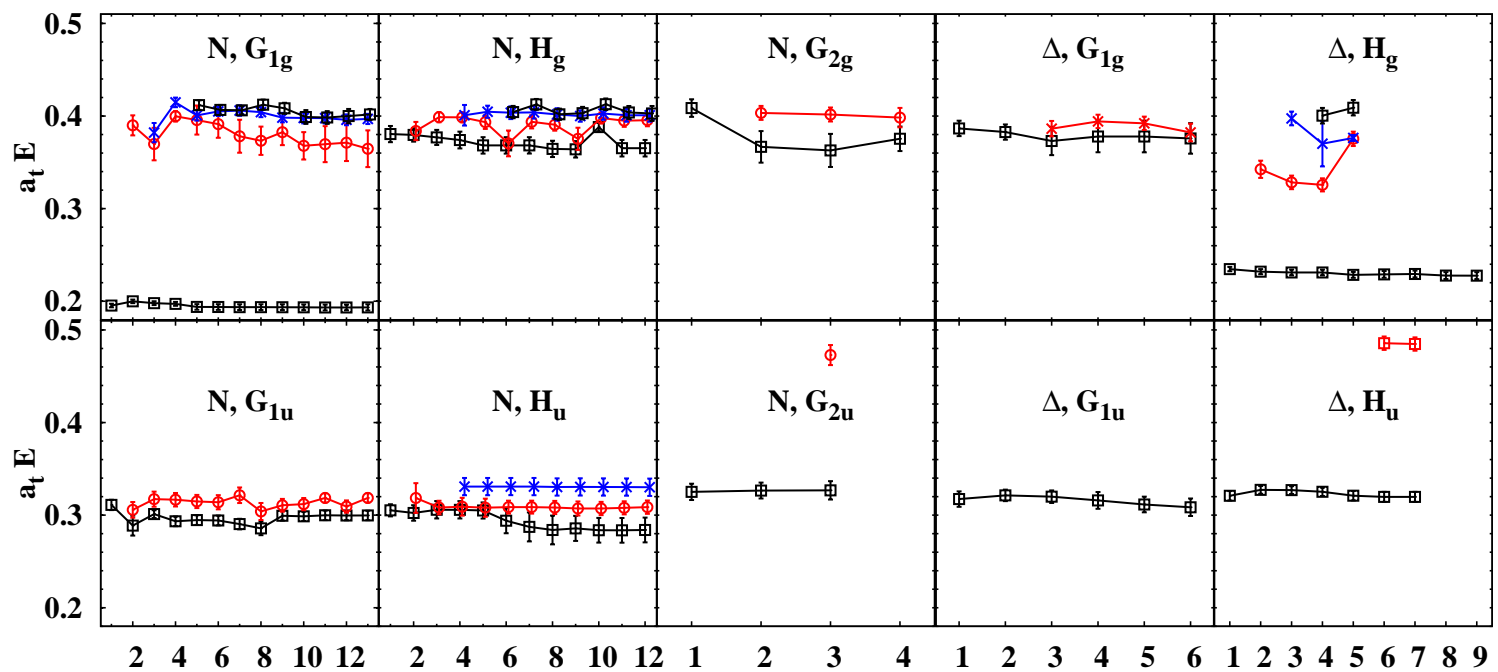

Figure 1: Effective masses in lattice units vs. dimension of matrices of correlation functions. Upper panels show the positive-parity channels and lower panels show the negative-parities channels. no matrices of correlation functions are obtained for the $\Delta, G_{2 g / u}$ irreps.

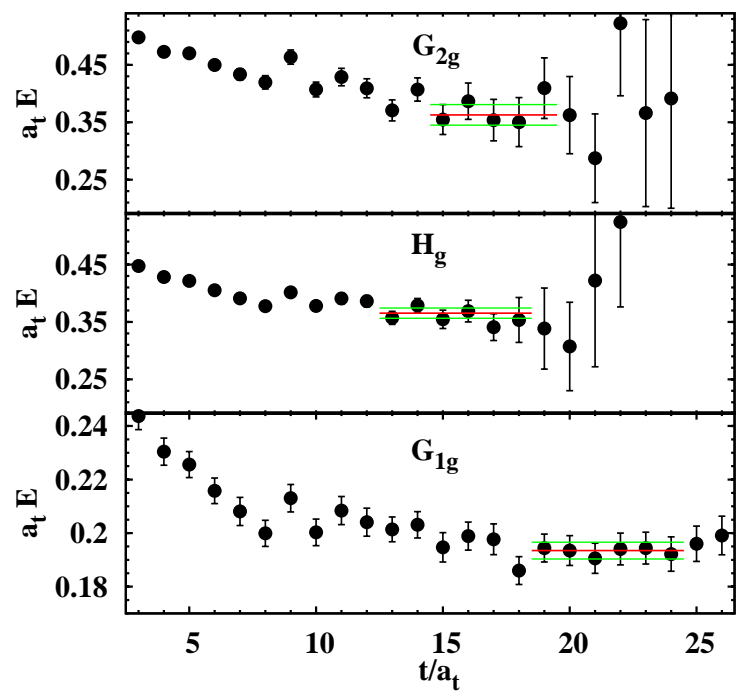

Figure 2: Effective energies for the lowest states in $G_{1 g}, H_{g}$ and $G_{2 g}$ irreps with isospin 1/2. Time ranges used for and energies obtained from fits are shown by horizontal lines. 


\subsection{Pattern of low-lying energies}

Figure 3 show the lowest energies from each irrep in isospin 1/2 and 3/2. Energies are converted to physical units. They are determined based the diagonalization of matrices of correlation functions consisting of the best sets of operators. This graph shows the pattern of level orderings for the different irreps, but it is not to compare the results with experimental values because our pion mass is too heavy and there may be significant discretization errors. Nevertheless, it is interesting to compare the pattern of level orderings with empirical data.

In the positive-parity channels, besides the nucleon ground state and the delta ground state, isospin $1 / 2, G_{2 g}$ and $H_{g}$ states are nearly degenerate within errors. If this degeneracy holds in the continuum limit and no degenerate partner exists in $G_{1 g}$, these two eigenstates correspond to the subduction of $J^{P}=5 / 2^{+}$. In our simulations we have no evidence for a $3 / 2^{+}$state that has lower energy than $5 / 2^{+}$state. Indeed in nature $N\left(5 / 2^{+}, 1675\right)$ is lighter than $N\left(3 / 2^{+}, 1705\right)$. Our results agree with this pattern, though our simulation conditions are far from physical. Previous lattice calculations that used $H_{g}\left(H_{u}\right)$ operators, such as Rarita-Schwinger projected operators, assumed that the lightest state corresponds to spin $3 / 2^{+}\left(3 / 2^{-}\right)$. Spin identification of the lowest $H$ state is uncertain unless $G_{2}$ irreps are included, i.e., the state could have spin $5 / 2$ or higher. The $G_{2}$ baryon operators cannot be constructed by local operators, therefore displaced operators are needed for the lattice simulations of excited baryon spectrum.

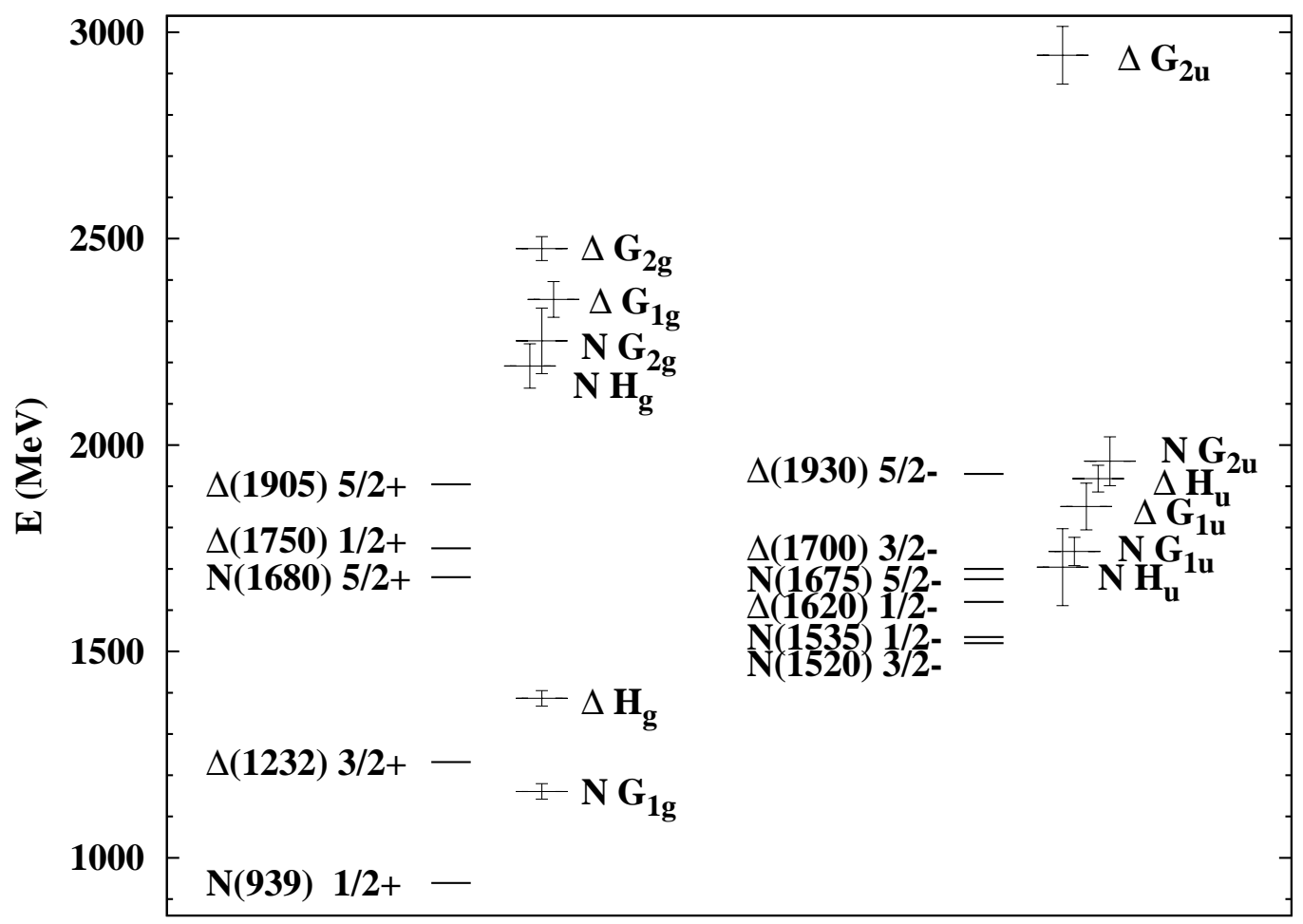

Figure 3: The energies obtained for each symmetry channel of isospin $1 / 2$ and $3 / 2$ baryons are shown. The energies are converted in $\mathrm{MeV}$ using the scale $a_{t}^{-1}=6.05 \mathrm{GeV}$. 
In the negative-parity channels, the level orderings from the lattice simulations go as follows: the $N^{*}, H_{u}$ corresponding to $J^{P}=3 / 2^{-}$(no degenerate partner with $N^{*}, G_{2 u}$ ) is the lowest, the $N^{*}, G_{1 u}$ corresponding to $1 / 2^{-}$is slightly above, the $\Delta^{*}, G_{1 u}$ corresponding to $1 / 2^{-}$is next, the $\Delta^{*}, H_{u}$ corresponding to $3 / 2^{-}$(no degenerate partner with $\Delta^{*}, G_{2 u}$ ) is slightly above, the $N^{*}, G_{2 u}$ corresponding to $5 / 2^{-}$is next and finally the $\Delta^{*}, G_{2 u}$ corresponding to $5 / 2^{-}$is the highest. These spin identifications are only tentative. In our simulations, we cannot find more that one operator in isospin $3 / 2, G_{2 g / u}$ channel, thus there is uncertainty in these channels. The pattern of level orderings in the negative-parity channels is consistent with the experimental data, except for the ordering of the $\Delta^{*}, H_{u}$ and the $N^{*}, G_{2 u}$ states.

It is intriguing to note that comparison of energies between different parities gives very different conclusions. For instance, the (lowest) $\Delta^{*}, 3 / 2^{-}$state should appear between the $N^{*}, 5 / 2^{+}$and the $\Delta^{*}, 1 / 2^{+}$, but our calculation does not agree with this pattern. We do not find a positive-parity excited state that has lighter energy than the lowest negative-parity state in isospin $1 / 2$. Further studies of lattice simulations are needed to understand realistic mass gaps between parities.

For more details about the quenched baryon spectrum based on extended operators, readers are referred to Ref. [12].

This work was supported by the U.S. National Science Foundation under Award PHY-0354982 and by the U.S. Department of Energy under contracts DE-AC05-06OR23177 and DE-FG0293ER-40762.

\section{References}

[1] K. I. Ishikawa, Nucl. Phys. Proc. Suppl. 140, 20 (2005) [arXiv:hep-lat/0410050].

[2] L. Zhou and F. X. Lee, arXiv:hep-lat/0604023.

[3] T. Burch, C. Gattringer, L. Y. Glozman, C. Hagen, D. Hierl, C. B. Lang and A. Schafer, arXiv:hep-lat/0604019.

[4] K. Sasaki and S. Sasaki, PoS LAT2005, 060 (2005) [arXiv:hep-lat/0508026].

[5] K. Sasaki and S. Sasaki, Phys. Rev. D 72, 034502 (2005) [arXiv:hep-lat/0503026].

[6] S. Basak et al. [LHP Collaboration], Nucl. Phys. Proc. Suppl. 140, 278 (2005) [arXiv:hep-lat/0409082].

[7] J. M. Zanotti, D. B. Leinweber, A. G. Williams, J. B. Zhang, W. Melnitchouk and S. Choe [CSSM Lattice collaboration], Phys. Rev. D 68, 054506 (2003) [arXiv:hep-lat/0304001].

[8] S. Basak et al. [Lattice Hadron Physics Collaboration (LHPC)], Phys. Rev. D 72, 074501 (2005) [arXiv:hep-lat/0508018].

[9] S. Basak et al., Phys. Rev. D 72, 094506 (2005) [arXiv:hep-lat/0506029].

[10] M. Luscher and U. Wolff, Nucl. Phys. B 339, 222 (1990).

[11] T. R. Klassen, Nucl. Phys. B 533, 557 (1998) [arXiv:hep-lat/9803010].

[12] A. Lichtl, arXiv:hep-lat/0609019. 